\title{
"BUSCANDO VIDA...": HACIA UNA TEORÍA AYMARA DE LA DIVISIÓN DEL TRABAJO POR GÉNERO
}

\author{
“SEEKING FOR LIFE...”: TOWARDS A THEORY ON AYMARA \\ GENDER LABOR DIVISION
}

\author{
Vivian Gavilán Vega*
}

\begin{abstract}
El propósito de este artículo es describir e interpretar la forma en que la población aymara del altiplano del norte de Chile piensa y practica la división del trabajo por género. Me interesa analizar las relaciones que establecen mujeres y hombres dentro de los hogares y conocer la teoría aymara de la división del trabajo y de la diferencia sexual. A través de la descripción y análisis de la distribución y valoración del trabajo entre las distintas categorías aymara del ciclo vital de los integrantes del hogar, sostengo que se dan relaciones de intercambio que conducen a desigualdades sociales más que a un intercambio de equivalentes. Sin embargo, los contenidos de estas desigualdades adquieren características particulares, tanto por los efectos de la inserción de las comunidades campesinas en la estructura socioeconómica regional como por las ideologías de género que forman parte de las prácticas productivas y reproductivas de la comunidad aymara.
\end{abstract}

Palabras claves: Género, comunidades aymara, norte de Chile.

The purpose of this study is to describe and explain how the Aymara population of the highlands of Northern Chile conceptualise and practice a division of labour by gender. I intend to analyze the relationship established by women and men in the home, and interpret the Aymara theory concerning the division of labour and sexual differentiation. By describing and analyzing the distribution and evaluation of labour among the different Aymara social categories in the life cycle of household members, I assert that exchange relationships generate social inequalities rather than egalitarian interchange. These inequalities acquire particular characteristics as a consequence of the insertion of peasant communities in the regional socio-economic structure, as well as of the gender ideology that constitutes part of the reproductive practices of the Aymara community.

Key words: Gender, Aymara communities, northern Chile.

\begin{abstract}
"Yo voy a trabajar, voy a buscar vida, ayuda pa' mis hijos, voy a covar eras a Chiapa, Camiña; pasteo a medias, vendo en la feria. Así ya tengo cómo mantener la familia." (Testimonio de Juana M.; Cariquima).
\end{abstract}

Los estudios sobre las comunidades aymara del norte chileno han prestado poca atención a las condiciones de la familia campesina e indígena, a las relaciones sociales que se dan dentro de ella y en especial a la división del trabajo por género. Durante las últimas décadas la mayor parte de los trabajos en estas y otras comunidades andinas han representado las relaciones entre mujeres y hombres en el hogar en términos complementarios, en que ambos géneros contribuyen a la reproducción social de la familia. Las normas y valores, propiamente andinos, determinarían niveles de flexibilidad que conducirían a enfatizar más en la inclusión que la exclusión y a no dicotomizar entre los roles, ni basarse en teorías discriminatorias (Anderson 1990; Casos 1990; De la Cadena 1985 y 1992; Harris 1980, 1985, entre otros).

Sin embargo, otro conjunto de estudios habla de prácticas discriminatorias que indicarían diferenciación y subordinación de las mujeres a los hombres (Deere 1992; Stolen 1987; Warren y Bourque 1976). A pesar de estas importantes contribuciones, la cuestión de la distribución del trabajo familiar y la explicación indígena de la misma, es aún un tema por investigar.

Los pocos estudios existentes para el norte chileno que apoyan la hipótesis de la complementariedad entre mujeres y hombres en la familia altiplánica adoptan una postura más esencialista de la sociedad y cultura aymara. Se asume que la relación mujer-hombre en la unidad doméstica se basa en un modelo complementario, igualitario o jerarquizado, cuyo origen lo hallamos en la persistencia de una matriz cultural andina. Esta matriz, basada en un pensamiento dual binario, deter-

* Taller de Estudios Andinos, Borgoño 85, Arica. teandinos@ 123mail.cl 
minaría relaciones de complementariedad entre opuestos apoyadas por relaciones de reciprocidad permanente (Flores 1996; Pizarro 1987; Van Kessel 1992). Así, las prácticas discriminatorias y la asimetría en las relaciones de género que se observan en la actualidad se atribuyen al proceso de transformaciones provocadas por la sociedad nacional y a la expansión capitalista. Este enfoque se basa en la existencia de un pasado no contaminado; es decir, parte del supuesto que antes de los distintos procesos de invasión y colonización habrían existido relaciones equilibradas e igualitarias. A pesar de la posible utilidad de esta tesis como marco de referencia, la cuestión de la complementariedad y simetría en la unidad conyugal se ha asumido más como una realidad de hecho que como una hipótesis a contrastar, lo que ha entorpecido las investigaciones empíricas, y en consecuencia, las relaciones al interior del hogar no han sido investigadas.

Los trabajos, cuya perspectiva histórica se opone a la anterior, han generado un interesante debate en torno a la cuestión campesina e identidad étnica del pueblo aymara (Carrasco 1993, 1994; González 1990; González y Gundermann 1989; Gundermann 1986). Estos llaman la atención en la necesidad de poner énfasis en el estudio de las dinámicas socioeconómicas y sociopolíticas actuales de las familias aymara, en el contexto de sus relaciones con la sociedad nacional. Sugieren que las comunidades han estado vinculadas históricamente al mercado y al Estado, pero que los procesos de mercantilización se acentuaron a partir de la década del 60, provocando una marcada diferenciación social del campesinado. Proponen, por otra parte, que a pesar de las transformaciones los aymara continúan constituyendo un grupo étnico diferenciado tanto por sus propias estrategias de vida como por la sociedad regional y nacional. Ciertamente, los avances en este sentido son valiosos; no obstante, el estudio de la situación actual del campesinado ha obviado la cuestión de la división del trabajo familiar y la concepción indígena de éste. En este sentido, han aplicado las categorías analíticas válidas para el estudio de la familia y el hogar en sectores no indígenas de Chile. Lo que ha supuesto asimilar las ideas existentes en nuestro contexto cultural a la realidad aymara, dejando fuera una parte clave en la comprensión de la realidad cultural y socioeconómica de este pueblo indígena.
En este contexto, en este artículo me interesa explorar en la organización del trabajo familiar en la zona altiplánica de la Primera Región desde un enfoque de género. Intento describir e interpretar la asignación diferencial del trabajo entre los integrantes de los hogares, particularmente a través de sus ciclos vitales, para reflexionar en torno a las transformaciones que han ocurrido al interior de la unidad doméstica.

Las preguntas que formulo son las siguientes: ¿Cómo se distribuye el trabajo entre los integrantes de la unidad doméstica?, ¿cuáles son las tareas apropiadas y no apropiadas para mujeres y hombres?, ¿cuáles son las explicaciones de mujeres y hombres para justificar la asignación, el ritmo y la intensidad diferencial del trabajo entre los géneros?, ¿cuáles son las valoraciones que tienen las actividades?, ¿cómo se relaciona la inversión de trabajo, el acceso a los medios de producción y la distribución y consumo del producto con el estatus de mujeres y hombres?, la división el trabajo por género, ¿es un operador que permite o facilita desigualdades sociales?

Para abordar estas interrogantes recurro al análisis de género, el que conceptualiza a la unidad doméstica y a la familia como un espacio de reproducción de la fuerza de trabajo. La recopilación de información empírica la he obtenido a través de quince entrevistas a mujeres y hombres solteros y casados, doce historias de vida de mujeres de distintas edades y dos de hombres, realizadas en el marco de dos proyectos en curso financiados por FONDECYT; y también de datos recolectados en trabajo de campo realizado en etapas sucesivas desde el año 1982 en el altiplano sur y norte de la Región de Tarapacá. El trabajo etnográfico más importante lo realicé en las comunidades de Isluga y Cariquima entre enero de 1982 a agosto de 1984. Después he permanecido por temporadas cortas en las comunidades y he trabajado con familias migrantes en la ciudad. En este período se han producido cambios que no he registrado en su totalidad, por lo que las descripciones de las actividades que realizan mujeres y hombres pueden haber sufrido variaciones que no destaco. En este sentido, los datos que expondré presentan limitaciones para dar cuenta de las transformaciones. Sin embargo, éstos, junto con la información obtenida en fuentes secundarias, permiten una aproximación a las respuestas que me formulo en este artículo. 


\section{Las Categorías de Análisis Usadas en este Estudio}

Los diversos estudios sobre la división sexual del trabajo que han aplicado un enfoque de género han demostrado que lo que se considera trabajo doméstico, trabajo productivo y reproductivo y su relación no es constante y que en muchos casos en la unidad doméstica se dan relaciones de intercambio y no de puesta en común, por lo que se hace necesario investigar y no darlas por sentadas. Así, el debate se construye alrededor de las definiciones del trabajo doméstico, trabajo, no-trabajo, valores de uso y valores de cambio, relación entre actividades productivas y reproductivas, entre otros (Deere 1992; Harris 1986; Harris y Young 1981; Mackintosh 1979; Moore 1990).

Al enfrentar el estudio de la división del trabajo familiar entre los aymara del altiplano, he procedido a describir las prácticas e ideas aymara en torno a las actividades diarias desarrolladas por todos los miembros de la unidad doméstica. Sigo a Deere (1992: 19) al conceptualizar a la familia como un espacio de reproducción de la fuerza de trabajo. De otro lado, pienso, como afirma Mackintosh (1981: 7), que comprender la división sexual del trabajo implica examinar no sólo los trabajos que los hombres y las mujeres hacen, sino también las relaciones bajo las cuales éstas se realizan (ej. las relaciones sociales de producción).

Uno de los aspectos resaltantes que aporta la perspectiva de género al análisis social es la necesidad de ampliar la esfera económica para incluir la reproducción de mercancías tanto como la reproducción social de las personas. Esto implica intentar comprender cómo la reproducción de las personas y la regulación social de la sexualidad forman parte del mismo proceso de producción y por lo tanto -de la concepción materialista- de la economía política. El género y la sexualidad se ponen al servicio de la división sexual del trabajo, pero también el género normativo se pone al servicio de la reproducción de la familia normativa (Buttler 2000). En el altiplano, la familia nuclear o restringida es una unidad doméstica y un hogar, una unidad de producción y consumo y está conformada por la unidad conyugal y sus hijos. Sin embargo, los Siblings, hermanos y sus esposas e hijos, pueden ser una unidad de trabajo (ayuda mutua interfamiliar) y pueden ser una unidad de tenencia o manejo de recursos productivos (rebaños colectivos, administración conjunta de chacras, etc.). Estas unidades sociales conforman un grupo de patriparientes exogámicos que se localizan en una "estancia", localidades llamadas así y donde pueden residir uno o más grupos. Se trata de grupos corporados en torno a derechos de tierra localizados que no tienen un carácter permanente y se rigen por un principio patrilineal de pertenencia. Poseen una identidad sociopolítica y son los grupos socialmente más importantes de las congregaciones rituales locales. Varias "estancias" conforman una comunidad, unidad geográfica con territorio propio, que se protegen frente a otros y que se reproducen, también, en torno a fiestas patronales, carnavales y a través de las pautas matrimoniales (Gavilán 1995) ${ }^{1}$.

Sin duda, la ganadería es la actividad más importante. Al observar las tareas cotidianas del hogar, nos damos cuenta que, aparte de las exigencias que impone el cuidado de los animales, hay otro conjunto de actividades que se deben realizar para obtener los recursos social y culturalmente necesarios para la reproducción biológica, económica y social de la unidad doméstica. Con el objeto de describir las tareas que se realizan las he agrupado en cuatro grandes categorías: actividades productivas, para el intercambio, reproductivas, y otras actividades sociales. Las primeras, consideran cuatro tipos de labores relacionadas directamente con la producción campesina: ganadería, agricultura, textilería y cría de aves de corral. Las segundas tienen que ver con el intercambio tanto de productos como de trabajo, entre los que es importante distinguir si se realiza en el ámbito local o extralocal. Como tareas reproductivas, incluyo aquellas actividades que se realizan en la casa para la alimentación familiar, la crianza de los menores, aseo y lavado y también la elaboración de materiales para los ritos, tales como la preparación de chichas, algunos tejidos y otros. La cuarta categoría refiere a aquellas labores sociales que forman parte de la rutina de una familia altiplánica, tales como asistir a asambleas comunitarias, reuniones escolares o dirigidas por el municipio, entre otras. Para la descripción de la distribución de los medios de producción he distinguido aquellos bienes más importantes por tipo de actividad tanto productiva como reproductiva.

Esta organización para la descripción ha intentado seguir tanto las prácticas cotidianas como 
el discurso aymara respecto de lo que mujeres y hombres consideran como trabajo. Para el ordenamiento de la distribución de los medios de reproducción consideré el valor asignado a los bienes tanto a través de las entrevistas, pero especialmente a través de las prácticas hereditarias (testamentos y otros).

\section{Mujeres y Hombres Aymaras en la Reproducción de la Unidad Doméstica}

"Buscando vida" es una metáfora muy usada por mujeres y hombres para referirse a los esfuerzos desplegados para la reproducción económica y social de la familia. Esta ayuda a comprender el funcionamiento de la unidad doméstica y permite aproximarnos a la idea del trabajo en el contexto altiplánico aymara. Las estrategias son múltiples y deben ser generadas por las personas, como miembros de una unidad de producción y de consumo; pero, como en otras sociedades, la distribución, la intensidad y el ritmo de las tareas son asignadas diferencialmente según el género y la fase del ciclo vital de las mismas.

La economía de las comunidades del altiplano se basa en la ganadería de llamas y alpacas y en menor medida de ovinos. En la zona sur se agrega la agricultura de quinua y papas como segunda actividad productiva y sólo en algunas localidades con condiciones climáticas más favorables, el cultivo de ajo. La textilería va perdiendo relevancia debido a los efectos de la mercantilización; la introducción de artículos de reemplazo determinó un retroceso en la producción textil.

La producción es en parte consumida y en parte se destina al mercado para la adquisición de otros bienes de consumo. Donde es posible la obtención de excedentes, una fracción es destinada al ahorro. Debido al alto grado de consumo de productos de procedencia externa, tales como alimentos y otros, las unidades familiares generan diversas estrategias para conseguir los recursos monetarios. En este sentido, la tenencia de animales es crucial para comprender la conducta económica de las familias, ya que, a través de la venta de carne en valles y ciudades accederán al dinero necesario; pero también por la venta de fuerza de trabajo. En los últimos quince años, también los subsidios estatales forman parte importante de los ingresos.

La apropiación y el acceso a los principales factores productivos combinan formas colectivas e individuales. Las tierras de pastoreo (bofedales o jok'o y cerros o waña-zuni) son de propiedad colectiva (normalmente bajo la forma legal de sucesiones), sobre las que poseen derechos de usufructo todos los comuneros y comuneras miembros de la estancia. Pero éstas son controladas por los hombres, ya que las mujeres se trasladan a la comunidad del marido y las normas consuetudinarias determinan que éstas accedan a los pastales a través de sus padres y/o esposos. En cuanto a las tierras agrícolas, se observa un patrón de tenencia individual, siendo su acceso normalmente también reservado a los varones, aunque no es raro encontrar en este caso el reconocimiento de derechos a algunas mujeres. Puede ocurrir a veces que éstas obtengan pequeñas fracciones a través de herencias familiares. Pese a ello, la intervención de la ley chilena, que reconoce un tipo de herencia bilateral, puede producir alteraciones en este patrón. $\mathrm{La}$ tenencia de animales, por otra parte, da cuenta de un sistema de propiedad y herencia bilateral. Por lo general éstos son obsequiados a los niños, sin distinción de sexo, por sus mayores, variando el volumen de acuerdo al número de animales que posea la familia, así como de la cantidad y calidad de los pastos que tenga la comunidad a la cual pertenecen.

La edad o etapa del ciclo vital en la que se encuentren las personas es importante tanto para la organización del trabajo como también para comprender la distribución y el consumo. De acuerdo a las propias categorías aymara, las que se pueden identificar como relevantes son las siguientes: en el caso de los hombres, serán yocalla o niño (entre 5 y 12 años aproximadamente), wayna o joven (entre 12 y 18 años aproximadamente, etapa previa al matrimonio), chacha o esposo (varón que está a cargo de una unidad doméstica, aunque la edad cronológica no es relevante, puede abarcar entre los 18 y 60 años) y achichi o abuelo (varón que se halla en la fase final de su ciclo laboral, entre los 60 y 80 años). Para las mujeres son: imilla o niña (entre los 5 y los 12 años aproximadamente), tawajo o joven mujer (entre los 12 y los 18 años: etapa previa al matrimonio), warmi o mujer casada (mujer a cargo de una unidad doméstica entre los 18 y 60 años) y apache o abuela (mujer en la fase final del ciclo laboral, entre los 60 y 80 años). En este sentido, según el tipo de actividad cada integrante de la familia realizará oficios específicos. 


\section{Las Actividades Productivas: Ganadería, Agricultura y Textilería}

El trabajo de pastoreo difiere según las épocas del año; en el verano estival, noviembre a marzo, el cuidado de los animales es más intensivo por ser temporada de pariciones y crecimiento de los cultivos que deben ser vigilados; en el invierno, de abril a octubre, requieren de menor acompañamiento pues permanecen en los cerros. Es diferente, también, si se trata de llamas o alpacas y ovejas. Las primeras, mejor adaptadas a las condiciones climáticas y topográficas, necesitan menos horas de trabajo, mientras que el ganado ovino requiere mayor tiempo de dedicación.

El pastoreo supone acompañar a los animales en la búsqueda de pastos y agua, reunirlos, atenderles en el momento de las pariciones y en caso de enfermedades. El cuidado de llamas y alpacas es realizado principalmente por las niñas jóvenes, imilla y tawajo, las mujeres casadas, los abuelos y los varones casados. Los niños y niñas por lo general no lo hacen (salvo como acompañantes), y los waynas sólo ocasionalmente lo realizan. Esto cambia en el caso de los ovinos pues son las imillas, los yocallas y las warmis quienes preferentemente asumen esta tarea. A veces pueden pastear las jóvenes, pero nunca los wayna, chacha y achichi. Los remedios caseros que se hacen para el tratamiento de algunas enfermedades son preparados y aplicados preferentemente por las mujeres adultas, aunque también por los hombres casados y abuelos; pero los farmoquímicos que se comienzan a introducir son aplicados en general por los varones casados.

La limpieza de canales que se realiza para el regadío de los bofedales y el baño que se les hace a los animales para prevenir los parásitos es una actividad colectiva (faena) donde participan los matrimonios: los hombres en los canales y las mujeres en la cocina, aunque éstas no se excluyen del todo; participan también como ayudantes en la toma de los animales. En los casos de viudas o mujeres solteras éstas deben preocuparse de enviar a uno de sus hijos mayores, contratar los servicios de un pariente o bien participar ella misma.

Así, vemos que el trabajo difiere si se trata de camélidos y de ovinos. En el caso de las llamas y alpacas no hay exclusiones por género, sí las hay de acuerdo a la edad. Sin embargo, se podría afirmar que los hombres, en las categorías jóvenes y adultos, no pastean ovejas, excepto los niños. El cuidado constante y el acompañamiento permanente constituyen una tarea asignada principalmente a la mujer, en especial a las madres, quienes reemplazan a los menores cuando éstos cumplen sus obligaciones escolares. Llama la atención que sea una de las pocas labores de la ganadería que los hombres jóvenes y adultos no realizan. Por otra parte, es importante mencionar que, dado el asalariamiento temporal de los hombres y/o la orientación de éstos hacia el comercio y el transporte, son las mujeres-esposas quienes se encargan del cuidado de los rebaños.

Las actividades agrícolas son enfatizadas en uno u otro género. La preparación de las chacras es efectuada principalmente por los hombres casados, participan menos las mujeres casadas, los varones jóvenes y los abuelos; lo mismo ocurre con el riego. En la siembra, aparecen nuevamente las mujeres, con excepción de las abuelas que lo hacen menos. Los varones también participan, particularmente en su condición de esposos. El "aporcar" (fertilizar), especialmente después de una lluvia y cuando la planta ya ha crecido, puede realizarse por ambos. Todos colaboran en la cosecha, aunque los niños y los jóvenes de ambos sexos sólo en ocasiones, debido a las actividades escolares². El almacenamiento es un deber de ambos cónyuges, pero el cuidado de éste está asociado a la mujer y a lo femenino ya que en general el consumo alimenticio de la familia es una preocupación especialmente de la warmi o esposa. La crianza de gallinas y a veces conejos es una preocupación de la warmi o los niños o niñas, aunque también lo pueden hacer los varones de la casa, un poco menos los wayna.

En la textilería se distinguen la fase de esquila, hilado y torcido, madejado y trenzado o tejido. Estos últimos a su vez se diferencian según sean de telar horizontal y a pedales. De acuerdo a sus funciones podemos identificar la vestimenta, los tejidos utilitarios y los ceremoniales. La esquila de animales, el hilado, torcido y madejado es una tarea que puede ser realizada por hombres y mujeres; pero son estas últimas las especialistas del hilado fino que se requiere para las prendas de uso ritual. Los varones jóvenes son los únicos que no practican el hilado, salvo en ocasiones. Sin embargo, la preparación de los hilos para los trenzados (mismir) y la confección de la cordelería es una actividad exclusiva de los hombres. Lo son tam- 
bién las telas (wayeta y cordillate) que se obtienen del telar a pedales. Son los esposos y hoy especialmente los abuelos quienes las tejen para venderlas como tela, para confeccionar pantalones de varones y las enaguas femeninas o cotona. Pero tanto lo primero como lo segundo hoy casi no se practica. Tal vez una forma de reemplazo ha sido el que los hombres compren a sus esposas faldas en los mercados urbanos, como regalos. Todos los demás tejidos, tales como bolsas de almacenamiento de los alimentos (costales), frazadas (iquiñas o panamanta), vestido femenino (hurku), mantas (hawayus) y otros usados en los ritos (vistallas, inkuñas) son realizados por las imillas, tawajo, warmi y apache. Es en esta actividad donde más se observan los efectos de la mercantilización, ya que cada vez las telas son reemplazadas por las industriales. Sólo cuando ésta tiene la posibilidad de convertirse en artesanía para la venta se realiza. Estos cambios son significativos en la situación femenina, ya que el tejido es una tarea que se asocia a las mujeres y a sus cualidades como sujeto social; no sucede lo mismo con la cordelería y los hombres. Aunque es una habilidad masculina, no está asociada al estereotipo masculino como lo es la música.

El tejido en agujas no se ha difundido tanto entre las mujeres de la zona sur. En Isluga, quienes se apropiaron de esta técnica fueron los hombres y no las mujeres. Asimismo, la confección de ropa y costura en máquina de coser manual era una tarea preferentemente masculina.

\section{Actividades de Intercambio}

Las actividades comerciales se desarrollan a nivel local y extralocal (valles o ciudades). La venta de carne y lana en el altiplano, por ejemplo, puede ser oficiada por hombres y mujeres casadas, junto con los abuelos. Por lo general los y las jóvenes no venden sus animales debido a la necesidad de formar los hatos para su futuro. El intercambio fuera de la comunidad es practicado por los varones especialmente casados y jóvenes. Los productos agrícolas son principalmente para el autoconsumo, pero si se intercambia una parte, esto se hace en el ámbito local por dinero o por otro producto. El ajo es comercializado por los varones y en algunas ocasiones por el matrimonio. La venta de tejidos, cuando se realiza a escala local, lo hacen las mujeres y si es fuera de la comunidad son los hombres quienes hacen de intermediarios. Esta tendencia ha variado debido al mayor contacto que han tenido las mujeres con los mercados regionales, especialmente por la intervención de programas de desarrollo desde el Estado (Centros de Madres) o de Organizaciones no Gubernamentales. Cuando ocasionalmente se venden los animales menores (gallinas o conejos), son los propietarios quienes lo hacen directamente, ya sea los niños/as o mujeres.

El comercio menor a escala local, especialmente de mercaderías, es realizado por el matrimonio, pero también por los jóvenes y en los últimos años son los wayna quienes optan por esta actividad. Cuando se trata de comercio mayor o contrabando fronterizo (desde las ciudades de Arica e Iquique hasta Oruro-La Paz o viceversa), los hombres casados son los principales actores.

Esta actividad hace que las familias tengan una gran movilidad. Se agregan a ello los movimientos migratorios hacia los valles en busca de pastos para el ganado, de trabajo o de productos agrícolas para el consumo; pero quienes participan particularmente en los viajes son los hombres, ya que son éstos los que mejor manejan las relaciones con el mercado y en general con la sociedad chilena ${ }^{3}$.

En el intercambio de trabajo se debe distinguir si se trata de asalariamiento, por productos o por trabajo y si se realiza en el mismo altiplano, en los valles o en la ciudad. En el primero, las mujeres pueden participar cuando son jóvenes de manera temporal en los valles como jornaleras, domésticas o como tejedoras. En la ciudad, son igualmente las tawajo quienes se emplean como asesoras del hogar. Los varones jóvenes y casados pueden emplearse en el altiplano en trabajos ordenados por el municipio o en mineras, en el valle como jornaleros y en la ciudad como obreros.

El trabajo por productos es cada vez menos frecuente en el altiplano y valles y es efectuado tanto por niños y niñas, jóvenes y casados ${ }^{4}$. En este caso se puede trabajar horas, días o meses generalmente en las tareas agrícolas y ganaderas, por lana, quinua o papa en el altiplano y maíz en los valles. El sistema de medierías (trabajo "al parti'o") es bastante frecuente en las familias con pocos animales. Este es solicitado generalmente por la pareja conyugal, el hombre o la mujer, pero el pastoreo es un trabajo del grupo doméstico. Los varones jóvenes pueden también asumir iniciativas de este tipo. 
El trabajo en medias se practica igualmente en los valles, donde el producto agrícola que se obtenga se reparte entre el dueño de la chacra y los arrenderos. En estos casos, las familias pueden continuar con las actividades del altiplano, dejando en medias a sus animales o a cargo de parientes o peones que pueden provenir de comunidades vecinas de Bolivia. Lo mismo ocurre con las chacras; pero cuando logran comprar tierras en los valles, van dejando, en un proceso paulatino, las actividades en su lugar de origen. Sin embargo, los animales y las obligaciones sociales continúan siendo asumidas.

El trabajo por trabajo se realiza entre las familias del mismo altiplano. La parentela establece un conjunto de contratos que se distinguen por el carácter de la devolución. Aunque éste también se va perdiendo, el que más se practica es "la turna" o "ayni" ya sea en las tareas de pastoreo o agrícolas. Se trata de trabajar una cantidad de horas o días las o los que deben ser devueltos en otra ocasión al solicitante de la misma forma. Aquí, son principalmente los representantes de la unidad doméstica quienes lo solicitan, pero son los jóvenes, matrimonio y abuelos quienes lo realizan.

\section{Las Actividades Reproductivas}

Las actividades domésticas se realizan en torno a la casa. Incluye tareas como la preparación de alimentos, el acarreo de agua y leña, el aseo, el cuidado de los niños y de los enfermos, y la preparación de los ritos. Identifico en éstas también la construcción de casas o arreglos de techumbres. La cocina es una preocupación de las warmi y abuelas, menos de imillas y tawajos. De manera más restringida los hombres también participan cuando son casados y ancianos. Beneficiar quinua y obtener los diferentes subproductos es una tarea principalmente femenina ${ }^{5}$, mientras que en el secado de papas (choqu'e chuñu) y carne (charqui) pueden participar ambos, con excepción de niños y varones jóvenes, aunque es también una labor especialmente de las mujeres. El beneficio de la quinua es particularmente importante para el consumo del hogar, ya que junto al maíz es la base de la alimentación. El acarreo de agua es una actividad de los niños y niñas, tawajo y warmi. La recolección de leña es más bien preocupación del matrimonio, salvo cuando ésta se hace en los cerros cercanos en la que pueden ir los niños.
El aseo y orden de la casa es responsabilidad de la mujer, pero el lavado de la ropa es realizado por cada uno de los integrantes de la familia. Aunque son las madres quienes se preocupan del lavado de los menores ${ }^{6}$. El cuidado de los niños es una tarea femenina, pero las tawajo pueden exceptuarse, pues se les permite invertir en actividades para su propio beneficio. En ocasiones también lo hacen los varones casados y abuelos. La atención de los enfermos es realizada generalmente por las mujeres. Si se trata de enfermedades complicadas será la pareja conyugal y si hay que trasladarse a otros pueblos o a la ciudad son los hombres los que deben ir en busca de curanderos o remedios. La preparación de los diversos elementos de los ritos ceremoniales es una actividad de las mujeres, especialmente de las casadas y las abuelas; pero los varones casados y los ancianos también se ocupan.

La construcción de las casas es una tarea de los hombres adultos, aunque no se excluyen del todo las mujeres. Los jóvenes normalmente construyen sus casas antes del matrimonio con la ayuda de sus padres, tíos y abuelos. Los arreglos de los techos también los realizan preferentemente los hombres; pero como esta tarea va acompañada normalmente de fiestas ceremoniales, las mujeres cocinan y acompañan en los rituales.

\section{Las Actividades Sociales}

Las actividades sociales se organizan en torno a la escuela, la comunidad, la iglesia y el municipio. En el primer caso, son principalmente los varones quienes se desempeñan como apoderados y las madres colaboran ocasionalmente. La comunidad impone la obligación de asistir a las asambleas comunales, donde se deciden las soluciones a los problemas que involucran a todas las unidades domésticas. Aquí, cada una de éstas debe tener un representante, éste es el chacha o varón casado. En su ausencia asistirá la mujer, sin mucha participación, excepto las viudas. El municipio es el que dirige las actividades de las organizaciones funcionales, tales como las juntas de vecinos y los centros de madres. En el primer caso, se asemejan a las asambleas comunales, en el segundo son las mujeres, preferentemente las casadas; pero los hombres generalmente están presentes y participan en las decisiones. Otras actividades municipales como conseguir los subsidios estatales u otros 
beneficios son principalmente hechas por los varones casados, aun cuando las mujeres también pueden intervenir.

Las personas adscritas a la Iglesia Evangélica Pentecostal tienen más exigencias que quienes adhieren a la Iglesia Católica. La primera convoca a varias reuniones en la semana y una vez al mes o más deben participar en salidas a predicar. Estos también asisten a reuniones de tres o cuatro días a otros pueblos. Los católicos se reúnen ocasionalmente a propósito de la visita de un cura y especialmente en las fiestas patronales y carnavales.

\section{Distribución del Trabajo en la Unidad Doméstica}

En esta descripción registré un total de 47 tareas. Desde una óptica cualitativa y con el objetivo de tener una aproximación a la distribución, opté por medir los grados de intensidad de trabajo que invierten los distintos integrantes de una unidad doméstica a partir de tres categorías: participan siempre o generalmente, participan ocasionalmente y no participan generalmente (Figura 1$)^{7}$.

En la Tabla 1 podemos ver la presencia o ausencia de hombres y mujeres en las distintas actividades identificadas. Estos datos muestran cómo las mujeres en su condición de esposa en plena productividad participan generalmente en 34 $(72,3 \%)$ de un total de 47 actividades identificadas; mientras que su contraparte masculina en 29 tareas $(61,7 \%)$. Siguen las tawajo y las apache con un total de 21 labores $(44,7 \%)$. La categoría masculina de achichi es la segunda en intensidad con 24 tareas realizadas generalmente (51\%). Las niñas están presentes en 16 actividades (34\%), mientras que los niños lo hacen en 7 tareas (3\%). Los wayna u hombres jóvenes, por lo general, están en 4 actividades $(8,5 \%)$.

En el otro extremo, es decir por lo general no participan, las warmi se exceptúan de sólo tres labores $(6,4 \%)$, mientras que los chacha lo hacen en 12 actividades (26,5\%). Las jóvenes no realizan normalmente 18 tareas $(38,3 \%)$; mientras que los jóvenes no participan generalmente en 31 (66\%) labores.

Entre las actividades que realizan ocasionalmente, hallamos nuevamente a las mujeres en la categoría warmi en 10 actividades (22\%); mientras que los chacha realizan 12 tareas ocasionalmente (26\%).
Estos resultados nos ofrecen un panorama general de la distribución del trabajo y también de los cambios que se gestan a través de las generaciones en los géneros. Una primera observación que podemos hacer es que la organización del trabajo involucra a todos los miembros de la familia y que existe una distribución desigual entre los mismos. En segundo lugar, si asumimos la distinción productivo-reproductivo, vemos que si bien en lo primero son pocas las tareas que excluyen a uno u otro, lo segundo está preferentemente en manos femeninas. Hay que destacar, sin embargo, que el pastoreo de animales es fundamentalmente una actividad femenina. El cuidado del ganado ovino y la textilería están exclusivamente a cargo de las mujeres, pero también lo realizan los hombres cuando son menores. Recordemos que el pastoreo de ovejas requiere de mayor dedicación (mayor exigencia en horas de trabajo) y que es considerado no apropiado para los hombres jóvenes y adultos. A pesar de que el argumento aymara reconoce esta asignación casi exclusiva a las mujeres, parecen no asumir la diferencia en la inversión de tiempo en el matrimonio. De todas maneras, el trabajo de las mujeres en la ganadería es normalmente reconocido al momento de heredar sus animales. De otro lado, los hombres asumen casi de manera exclusiva la preparación de las chacras y el riego.

En tercer lugar, visualizamos una diferencia notable en la continuidad/discontinuidad de las tareas campesinas entre mujeres y hombres. En el caso de las primeras observamos ciertos niveles de coherencia a través del ciclo vital. Ellas se inician tempranamente en las tareas que cumplirán a lo largo de su vida. En los segundos hallamos saltos abruptos entre los jóvenes y los adultos. Su ausencia en las tareas productivas puede explicarse por los cambios producidos en las últimas décadas. Un elemento a considerar son los procesos migratorios temporales en los que se involucran, vividos fuertemente en la zona altiplánica norte después del Puerto Libre de Arica (década de los 60) y que ha dejado en algunos casos sólo a población anciana y a mujeres solas. La migración por motivos de estudio afecta más a los hombres que a las mujeres. Estas últimas pueden acudir por trabajo doméstico a la ciudad, actividad que interrumpen temporalmente por recarga de trabajo en su familia o bien para las fiestas y carnavales en el altiplano. Así, hallamos una clara diferencia entre los pro- 


\begin{tabular}{|c|c|c|c|c|c|c|c|c|}
\hline \multirow{2}{*}{ Actividades Productivas } & \multicolumn{4}{|c|}{ Mujer } & \multicolumn{4}{|c|}{ Hombre } \\
\hline & Imilla & Tawajo & Warmi & Apache & Yocalla & Wayna & Chacha & Achichi \\
\hline $\begin{array}{l}\text { 1. Ganadería } \\
\text { Ovejas } \\
\text { Camélidos }\end{array}$ & |IIIIII & 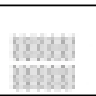 & 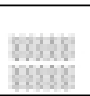 & $\approx$ & |IIIII| & $\approx$ & IIIIIII & $\approx \approx$ \\
\hline $\begin{array}{ll}\text { 2. } & \text { Agricultura } \\
\text { Preparación chacras } \\
\text { Siembra } \\
\text { Riego, limpieza canales } \\
\text { Cosecha } \\
\text { Guarda y almacenamiento }\end{array}$ & $\underset{\approx}{\approx}$ & $\approx$ & 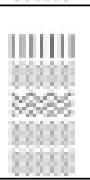 & $\underset{1 I I I I I}{\approx \approx}$ & $\underset{\sim}{\sim}$ & IIIIIII & 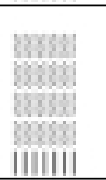 & $\frac{35328}{33238}$ \\
\hline 3. Cría de animales menores & Bande & Bnant & 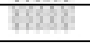 & $\approx$ & $\cos 2$ & $\approx$ & $\approx \infty$ & 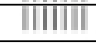 \\
\hline $\begin{array}{l}\text { 4. Textilería } \\
\text { Esquila } \\
\text { Hilado y torcido } \\
\text { Vestimenta } \\
\text { Utilitarios domésticos } \\
\text { Ceremoniales } \\
\text { Trenzados }\end{array}$ & 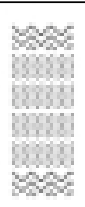 & 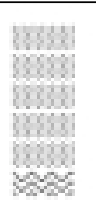 & 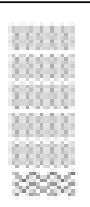 & $\underset{3}{I I I I I I}$ & $\underset{\text { IIIIIII }}{\sim}$ & $\underset{\text { IIIIIII }}{=}$ & $\underset{\text { IIIIIII }}{\approx}$ & 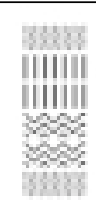 \\
\hline \multicolumn{9}{|l|}{ Actividades de Intercambio } \\
\hline $\begin{array}{l}\text { 1. } \text { Comercio } \\
\text { Ganadero Local } \\
\text { Ganadero Extralocal } \\
\text { Agrícola Local } \\
\text { Agrícola Extralocal } \\
\text { Tejido Local } \\
\text { Tejido Extralocal } \\
\text { Otros Productos de Mercado }\end{array}$ & $\approx$ & $\underset{\approx}{\approx} \approx$ & 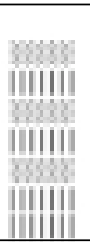 & $\begin{array}{l}30 x+4 \\
3030 \\
3\end{array}$ & $\approx$ & $\underset{\text { IIIIIIIII }}{\text { iIIII }}$ & 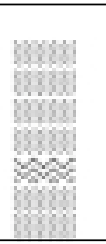 & 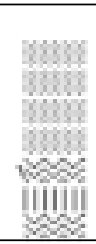 \\
\hline $\begin{array}{ll}\text { 2. } & \text { Trabajo } \\
\text { Asalariado en altiplano } \\
\text { Asalariado en valles } \\
\text { Asalariado en ciudad } \\
\text { Por productos en altiplano } \\
\text { Por productos en valles }\end{array}$ & 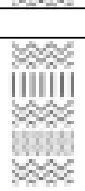 & 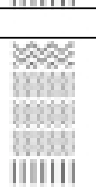 & $\begin{array}{l}\text { IIIIIII } \\
\frac{111 I I I I}{4}\end{array}$ & $\underset{\approx}{\approx} \approx$ & IIIIIIIII & 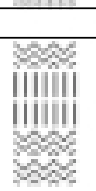 & 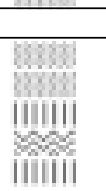 & 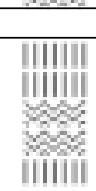 \\
\hline \multicolumn{9}{|l|}{ Actividades Reproductivas } \\
\hline $\begin{array}{l}\text { 1. Trabajo doméstico } \\
\text { Preparación alimentos } \\
\text { (Molienda de granos) } \\
\text { Cocina } \\
\text { Crianza } \\
\text { Aseo casa } \\
\text { Lavado } \\
\text { Acopio leña } \\
\text { Acopio agua } \\
\text { Preparación ritos } \\
\text { Construcción casas }\end{array}$ & $\begin{array}{l}\text { IIIIIII } \\
\text { IIIIIII } \\
\text { IIIIII }\end{array}$ & 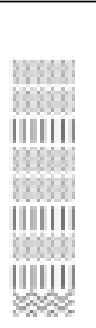 & 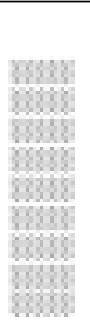 & $\begin{array}{l}\text { IIIIIII } \\
\text { IIIIIII } \\
\text { IIIIIII }\end{array}$ & 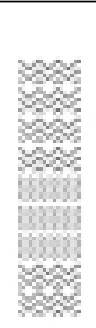 & $\underset{1 I I I I I}{\approx}$ & 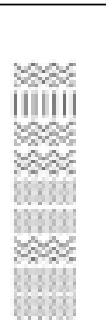 & 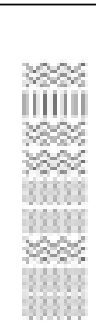 \\
\hline \multicolumn{9}{|l|}{ Participación Actividades Sociales } \\
\hline 1. Escuela (Centro de Apoderados) & $\approx$ & $\approx$ & III!!! & $\approx$ & & $\cong$ & $8035 \frac{1}{2}$ & $\approx \approx$ \\
\hline $\begin{array}{l}\text { 2. Comunidad: Asambleas } \\
\text { Centros de Madres } \\
\text { Festividades comunales }\end{array}$ & $\approx$ & iiiiiII & 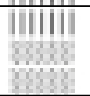 & $\underbrace{}_{0.1111}$ & $\approx$ & $\approx$ & 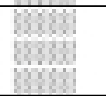 & 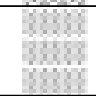 \\
\hline 3. Municipio & 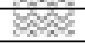 & $8 \times$ & $\|I\| I \|$ & \|\|\|\| & 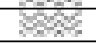 & 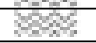 & 68808 & 38688 \\
\hline $\begin{array}{l}\text { Rituales } \\
\text { Bautizo } \\
\text { Corte de pelo } \\
\text { Matrimonio } \\
\text { Funerales } \\
\text { Fiestas Patronales de iglesia } \\
\text { Carnavales } \\
\text { Floreo }\end{array}$ & $\underset{\| \approx}{\approx}$ & $\begin{array}{l}\approx \\
\approx \\
\text { IIIIIIII }\end{array}$ & 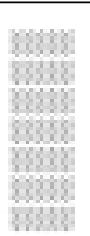 & 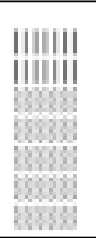 & 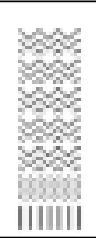 & $\begin{array}{l}\approx \approx \\
\approx\end{array}$ & 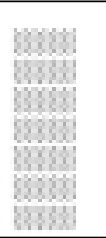 & 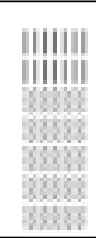 \\
\hline
\end{tabular}

sosid Siempre participa

$\approx$ Generalmente no participa

iIIIIII A veces u ocasionalmente participa

Figura 1. Actividades productivas y reproductivas, según fase del ciclo vital de los miembros de la unidad doméstica, por género. Productive and reproductive activities related to different phases of the life cycle of household's members, by gender. 
Tabla 1. Resultados actividades productivas y reproductivas por género y fase del ciclo vital. Productive and reproductive activities by gender and phase in the life cycle.

\begin{tabular}{l|rrrr|rrrr}
\hline \multirow{2}{*}{ Participación en Actividades } & \multicolumn{4}{|c|}{ Mujer/Fase Ciclo Vital } & \multicolumn{4}{c}{ Hombre/Fase Ciclo Vital } \\
\cline { 2 - 10 } & IImilla & Tawajo & Warmi & Apache & Yocalla & Wayna & Chacha & Achichi \\
\hline Siempre participan & 16 & 21 & 34 & 21 & 7 & 4 & 29 & 24 \\
Generalmente no participan & 25 & 18 & 3 & 17 & 33 & 31 & 12 & 12 \\
Ocacionalmente participan & 6 & 8 & 10 & 9 & 7 & 12 & 6 & 11 \\
\hline Total & 47 & 47 & 47 & 47 & 47 & 47 & 47 & 47 \\
\hline
\end{tabular}

yectos de vida de ambos géneros. Los padres y las madres han incentivado a los jóvenes a estudiar y a no realizar el trabajo campesino; mientras que a las mujeres las sacaban del colegio antes de terminar la enseñanza básica para que se ocuparan del ganado, tejido y otras actividades de preparación para el matrimonio ${ }^{8}$. En esta situación ha intervenido el sistema educacional del Estado chileno al no comprender las dinámicas culturales y de los ciclos laborales campesinos, al promover expectativas vinculadas a actividades urbanas y a desvalorizar las tareas agropecuarias. Ello sumado a una mayor mercantilización de la economía local que ha incentivado más a los hombres a la búsqueda de empleos, particularmente al comercio, contribuye a situar a los hombres jóvenes en un grave problema de discontinuidades y contradicciones; y a las mujeres a asumir el peso de la producción y reproducción de la unidad doméstica del altiplano?.

En cuarto lugar, los resultados muestran una clara concentración del trabajo en el matrimonio. La unidad chacha-warmi es fundamentalmente quien provee al hogar. El trabajo productivo femenino es tan reconocido como el del varón ${ }^{10}$. Esta participación es registrada por los censos nacionales de población, a pesar del subregistro actualmente existente: el 20,99\% de las mujeres rurales de la región de Tarapacá declaran estar trabajando. Mientras que la tasa de participación femenina en la zona rural del país sólo es de $11,82 \%$. El mismo patrón siguen las mujeres migrantes en la ciudad, ya que ellas realizan esfuerzos permanentes para contribuir a los ingresos familiares. Un estudio realizado en la comuna de Arica indica que ellas comienzan a trabajar remuneradamente a una edad promedio de 17 años, como jornaleras agrícolas o en empleo doméstico, siendo la actividad comercial por cuenta propia muy significativa. En términos de ingresos, las mujeres rurales entregan un aporte aproximado de un $41,2 \%$ a las entradas familiares (Carrasco 1994). Así, el rol de "proveedor" asignado exclusivamente a los hombres no operaría.

El trabajo reproductivo en manos de las mujeres marca otra diferencia. Ellas reconocen su contribución en las tareas domésticas, especialmente el cuidado de los hijos, actividad que cada vez es responsabilidad exclusiva de las mujeres; pero esta asignación es asumida como propia de las mujeres y no apropiadas para los hombres-maridos.

El peso que sobrelleva el matrimonio para el mantenimiento del hogar y particularmente la mujer, se expresa en conflictos permanentes en la pareja. Mientras las esposas reclaman las medidas de sus aportes en las tareas productivas y reproductivas, los hombres se justifican buscando opciones de empleo fuera de la comunidad o realizando trámites de representación sociopolítica.

\section{El Control de los Medios de Producción y de Subsistencia}

El género y la fase del ciclo vital de las personas intervienen directamente en la propiedad de los medios de producción y en la herencia (Figura 2). Se distingue también la propiedad colectiva e individual. Los pastales son un bien colectivo y quienes detentan los derechos exclusivos de uso son los hombres casados de la comunidad. La tierra agrícola, sin embargo, es de propiedad individual y preferentemente masculina, aunque las mujeres pueden heredar pequeñas chacras. Así, los abuelos tienen mayores niveles de intervención en la determinación de las herencias. Los hijos dependen de la decisión de los padres para recibir legalmente sus derechos de tierras. Algunas mujeres cuentan en sus testimonios que compartir el trabajo con los suegros durante la primera etapa de 


\begin{tabular}{|c|c|c|c|c|c|c|c|c|}
\hline \multirow{2}{*}{$\begin{array}{l}\text { Distribución de los } \\
\text { Medios de Producción } \\
\text { y Reproducción }\end{array}$} & \multicolumn{5}{|c|}{ Mujer } & \multicolumn{3}{|c|}{ Hombre } \\
\hline & Imilla & Tawajo & Warmi & Apache & Yocalla & Wayna & Chacha & Achichi \\
\hline $\begin{array}{l}\text { Ganadería } \\
\text { Pastales } \\
\text { Animales }\end{array}$ & & $3 \approx$ & $3 \approx$ & 38 & 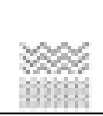 & $\approx \approx$ & & \\
\hline $\begin{array}{l}\text { Agricultura } \\
\text { Chacras } \\
\text { Semilla } \\
\text { Instrumentos trabajo } \\
\text { Aguas }\end{array}$ & $\underset{\mho \approx}{W}$ & $\underbrace{\| \approx}_{\approx \approx}$ & $\| \underset{\approx}{\approx}$ & 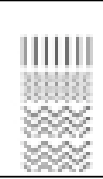 & $\underset{\approx \approx}{\approx \ldots}$ & $\| \widehat{\| \widehat{N}} \widehat{\|}$ & Das38: & \\
\hline $\begin{array}{l}\text { Textilería } \\
\text { Telar Horizontal } \\
\text { Telar pedar } \\
\text { Huso y otros } \\
\text { Lana }\end{array}$ & 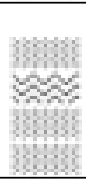 & 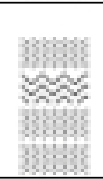 & 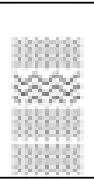 & 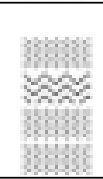 & 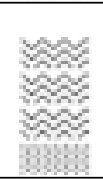 & 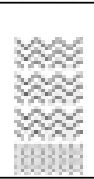 & $\underset{\approx \approx}{\| \approx}$ & $\approx$ \\
\hline Vehículos & & 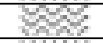 & 33033 & 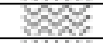 & $\$$ & \|\|$\|$ & & \\
\hline Capital comercial & $8 \%$ & \|\|$\|$ & \|\|$\|$ & \|\|$\|$ & 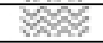 & & Z335:3 & \\
\hline $\begin{array}{l}\text { Utensilios domésticos } \\
\text { Vajilla } \\
\text { Piedra de moler } \\
\text { Tejidos almacenamiento } \\
\text { Ropa de cama } \\
\text { Otros tejidos } \\
\text { Objetos rituales } \\
\text { Instrumentos musicales }\end{array}$ & $\underset{33}{\approx \approx a}$ & 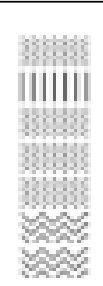 & 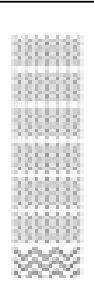 & 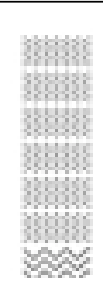 & 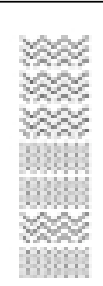 & $\underset{\approx}{\approx \ldots}$ & 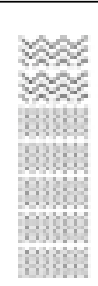 & 瓜 \\
\hline
\end{tabular}

Figura 2. Distribución de los medios de producción y reproducción según fase del ciclo vital.

Distribution of the mean of production and reproduction according to the phase of the life cycle.

casados es una obligación moral; como una forma de devolver los futuros derechos de acceso a pastales y chacras o como un modo de reciprocar la ayuda prestada para la independencia de la unidad doméstica. El agua que se usa para las chacras de regadío es colectiva y su uso entonces será determinado por los varones.

La filiación patrilineal, la patrilocalidad del matrimonio y la escasez de tierras hacen que las mujeres no accedan a este importante factor productivo. Los hombres aymara argumentan que éstas tienen acceso a través de su marido, quien tiene derechos en la comunidad de su padre. Asignarles a ellas implicaría conflictos entre familias y la fragmentación de la tierra al punto de hacer inviable la convivencia. Postulan que como una forma de compensarlas pueden heredar animales, tejidos y otros objetos.

Los animales pertenecen a ambos géneros en todas las categorías de edad. La ceremonia de corte de pelo es el momento en que los padres y padri- nos regalan los primeros ejemplares que se irán reproduciendo, junto con otros que van adquiriendo por regalo o compra hasta el matrimonio. Otra estrategia para conseguir ganado es a través del trabajo en medias, lo que implica que la mitad de las crías que se obtengan serán para el mediero. Si éste es un hombre o una mujer casada los animales serán para la unidad conyugal, aunque se tendrá presente quién invirtió mayor cantidad de trabajo. Los instrumentos de trabajo agrícola son principalmente objetos de propiedad masculina y son los varones casados y abuelos quienes deciden el uso familiar. La indumentaria textil es de propiedad individual y cada miembro de la familia será dueño de sus propios instrumentos.

Los artefactos domésticos son también de estricta propiedad individual. Tanto la vajilla, ollas y cubiertos, piedras de moler, las bolsas tejidas para el almacenamiento de los productos agrícolas, frazadas y camas de uso familiar son de la madre y abuela, mientras que aquellos que adquieren las 
tawajo por su propia cuenta son de su propiedad y no se usan, sino por ellas cuando forman su propio hogar. Las herramientas como carretillas, martillos e instrumentos musicales son de propiedad masculina. Las primeras son de hombres casados y los segundos tanto jóvenes como adultos son poseedores. Estos también son dueños de camas y frazadas que han recibido como obsequio de las madres.

Este régimen de propiedad individual se expresa también en el carácter bilateral del sistema de herencia. Mientras las mujeres heredan sus propias pertenencias tales como ganado, telares, hilos y tejidos, objetos de cocina, joyas, alimentos entre otros $^{11}$, los hombres transfieren los derechos de tierra agrícola, los instrumentos musicales, tejidos, ganado y herramientas en general. De esta manera, se identificaron ocho tipos de medios de producción y reproducción (Figura 2). El acceso a los medios de producción indica un estricto sistema de propiedad individual de algunos de los bienes socialmente necesarios para la producción y reproducción del hogar. En el caso de las tierras de pastoreo, si bien son de uso colectivo en la comunidad, legalmente son sucesiones que incluyen a varios hermanos de una estancia y comunidad, las que son transferidas legalmente al mayor de los hermanos.

Si distinguimos los medios de producción más relevantes en la economía del hogar, por ejemplo, la ganadería, agricultura, vehículos y capital comercial de aquellos que acompañan su reproducción, es decir, la textilería y tareas domésticas, vemos lo siguiente (Tabla 2).

De un total de ocho bienes de alto valor social, siete están en manos de los hombres-esposos, cifra que representa el $87,5 \%$; mientras que las mujeres-esposas acceden sólo al 37,5\%. Observamos una clara concentración en manos de los hombresesposos y abuelos. Si bien las mujeres y los jóvenes acceden a animales y en menor medida a chacras o tierras agrícolas, los pastales, terrenos para la agricultura y los instrumentos agrícolas son controlados por los hombres adultos.

De este modo y como ocurre con la distribución del trabajo, el sistema de propiedad establece una diferencia importante. La exclusión de las mujeres a la propiedad de la tierra sitúa a los hombres en una posición de poder y de privilegio, ya que les permite ejercer autoridad en las decisiones y controlar, hasta cierto punto, la relación en la unidad conyugal. Aun así, la distribución del producto bajo el concepto de propiedad individual otorga a las mujeres cierto control sobre su trabajo. En las familias que han migrado a la ciudad pueden tomar decisiones sobre el uso del dinero obtenido por su trabajo o de sus cuentas de ahorro. Es frecuente también que se lamenten cuando no puedan obtener ingresos independientes.

\section{La Distribución del Producto}

El destino del producto familiar se distribuye según sea para el consumo o ahorro de la unidad doméstica o para el gasto o ahorro personal. El gasto familiar está orientado al consumo de alimentos, ya sea su propia producción de carne, quinua y papas o adquiridos en el mercado como azúcar, fideos, arroz, vegetales y otros; de vestimenta; educación (útiles escolares o enviar a los varones jóvenes a la ciudad); herramientas de trabajo; al cumplimiento de las deudas sociales (cargos religiosos) y salud del matrimonio y su prole. En el caso de los jóvenes, el salario o producto que obtengan puede ser utilizado en parte para el consumo de la familia y en parte para su propio benefi-

Tabla 2. Distribución de los medios de producción (ganadería, agricultura, vehículos, capital comercial). Distribution of the mean of production (livestock, agriculture, vehicles, capital investment).

\begin{tabular}{l|cccc|cccc}
\hline \multirow{2}{*}{ Acceso a Medio de Producción } & \multicolumn{4}{|c|}{ Mujer } & \multicolumn{4}{c}{ Hombre } \\
\cline { 2 - 9 } & IImilla & Tawajo & Warmi & Apache & Yocalla & Wayna & Chacha & Achichi \\
\hline Propietarios & 1 & 1 & 3 & 2 & 1 & 2 & 8 & 6 \\
No propietarios & 7 & 5 & 3 & 4 & 7 & 3 & 0 & 2 \\
Tienen acceso, acceden pero poco & & 2 & 2 & 2 & & 3 & 8 & 8 \\
\hline Total & 8 & 8 & 8 & 8 & 8 & 8 & 8 \\
\hline
\end{tabular}


cio. El gasto personal se orienta hacia la adquisición de ropa, útiles escolares, útiles de cocina, camas, lana como materia prima, herramientas o para la diversión. El ahorro familiar, cuando es posible, se obtiene para la formación de un capital que sirva para emprender nuevos negocios o compra de animales. El personal, especialmente de los jóvenes, se constituye para la formación de un capital que les permita adquirir y acrecentar sus bienes para el futuro.

El consumo o venta de los animales y sus subproductos para la familia es decisión del chachawarmi o matrimonio. Aunque se tiene un registro estricto de si se trata de ejemplares de la mujer o el varón. Ambos deciden tanto en el tipo de animal que se sacrificará y en el tipo de orientación que se le dará al producto. Sin embargo, debido a que los hombres son los principales comercializadores éstos suelen hacer uso de parte del dinero en la ciudad en paseos, comiendo y bebiendo, en el que las mujeres no participan. Los animales de niños y jóvenes no se venden ni se consumen, pues es el capital con el que contará en su vida futura. La papa y quinua son principalmente para el autoconsumo y será la unidad conyugal quien la distribuya. Pero son las mujeres las encargadas por excelencia de su consumo. En aquellos pueblos donde se cultiva el ajo, su venta será también del matrimonio, aunque los varones aparecen con mayor capacidad de decisión sobre el dinero obtenido. Los jóvenes, ya sean mujeres u hombres, también pueden emprender algún tipo de actividad agrícola a través de sus padres y en este caso la mayor parte del producto será para su beneficio personal, el que podrá ser ocupado para la compra de bienes de consumo o bien para su ahorro personal.

La textilería: hilados, tejidos o trenzados, es de propiedad individual. Los menores pueden recibir regalos y cuando ellos mismos los confeccionan pueden decidir guardarlos para su propio uso, trocarlos o venderlos; si esto ocurre, el producto o dinero será para su propio beneficio. Lo mismo ocurre con los adultos, aunque en el caso de las personas casadas deben orientarlo al consumo o ahorro de la familia. Aun así, la unidad conyugal tiene claridad de cuánto contribuirá cada uno a los ingresos familiares.

En el caso de la adquisición de un vehículo, si se ha hecho con aportes de la warmi y el chacha, es de propiedad del matrimonio, pero su uso es preferentemente del varón. En pocas ocasiones ocurre que lo hagan los jóvenes: es más común que se asocien los hijos solteros con el padre y la madre o bien entre hermanos, lo que igualmente supone propiedad compartida en la medida de sus aportes.

\section{La División Social del Trabajo, el Estatus de Mujeres y de Hombres y las Ideologías de Género}

Hemos visto que tanto la división sexual del trabajo como la fase del ciclo vital de los miembros de una unidad doméstica son claves para comprender las dinámicas laborales que se dan al interior del hogar. Tanto el hombre-esposo como la mujer-esposa son jefes de una unidad doméstica o responsables directos de los procesos de trabajo y es en ellos en los que descansan la producción y reproducción de la misma. Al interior del matrimonio, son las mujeres quienes se encargan de la mayor parte de las tareas consideradas socialmente necesarias.

Observamos de este modo una clara diferenciación entre los géneros. Se distinguen las tareas reproductivas y algunas productivas. Las primeras son tareas apropiadas para las mujeres, mas no para los hombres. Entre las segundas, el trabajo de pastoreo es asignado a las mujeres. Según el pensamiento aymara el cuidado de las ovejas es una tarea de menor prestigio; así como todas las tareas campesinas se consideran de menor valor que el comercio y el transporte, actividades a las que tienden los hombres jóvenes. La valoración asignada al trabajo del ganado ovino se relaciona con los precios de mercado, pero también con ideas culturales asociadas a la reproducción simbólica de la familia y la comunidad, las cuales ubican a llamas y alpacas en un lugar esencial en los rituales propiciatorios.

Es notable que el discurso de mujeres y hombres insista en que cualquiera puede realizar los distintos tipos de trabajo y a la hora de las prácticas sea claramente diferenciado. Las explicaciones a estas diferencias se orientan a marcar "preferencias", mas no exclusiones. No obstante, estas preferencias se vinculan con las estrategias particulares desplegadas por cada unidad doméstica dirigidas también por las coyunturas por las que 
atraviesa. Es óptimo que las mujeres estén presentes en todas aquellas labores asociadas al consumo y a la fertilidad y abundancia, tales como la siembra, la cosecha, la alimentación, el pastoreo de animales (para su multiplicación). Lo femenino es fundamentalmente pensado como generativo y fértil. Mientras que lo masculino y los hombres se orientan más a las representaciones sociales y políticas.

Las actividades reproductivas y productivas en manos de las mujeres inciden en que éstas inviertan más trabajo. Los hombres así pueden disponer de mayores momentos de ocio, como jugar fútbol o divertirse con amigos y amigas. El ocio no es bien visto en las primeras. El ideal de mujer es aquella que siempre está pendiente del trabajo y su opuesto es ser "jaira warmi" (mujer floja), lo que es casi un insulto. Una mujer casada y joven es la que cuida y amamanta a sus guaguas mientras hila, teje y al mismo tiempo está pastoreando o vendiendo. Aquella que teje perfectamente y no se queja ni reclama. Un buen hombre-esposo también es un esforzado trabajador y cuida social y políticamente de su hogar. Estos estereotipos de género permiten justificar y reproducir la organización doméstica.

Es poco común ver a hombres y mujeres solas. Existen escasas rupturas matrimoniales y cuando enviudan vuelven a casar muy pronto. La vida en pareja se requiere para distribuir el trabajo, pero también para cumplir con los ideales de personas en esta y en la otra vida, después de muertos. El matrimonio es el rito de paso más importante. Este marca a las personas como miembros sociales de la comunidad, adquieren de este modo la condición de Jaque (gente) o adultos. Sin embargo, los recién casados son considerados como principiantes o guaguas, personas que comienzan a vivir en sociedad y por tanto hay que enseñarlos y dirigirlos. El comportamiento inicial de mujeres y hombres ilustra bien los modelos de esposa-esposo. Cuando la mujer llega a la casa de la familia del hombre, se entrega a la voluntad de la suegra y se esmera por cumplir de la mejor manera sus obligaciones que consisten en cuidar al ganado, beneficiar quinua y atender tanto a su marido como a sus nuevos parientes. Esta etapa dura dos años aproximadamente. La mayoría de las mujeres consideran que este período es muy difícil y no tienen descanso; sin embargo, ellas vuelven a repetir lo mismo cuando son mayores con las esposas de sus hijos. Para el hombre es igualmente difícil porque la norma y la moral exigen de él un comportamiento respetuoso de sus padres y de la familia de la mujer y porque normalmente está en medio de conflictos entre su esposa y su madre. Pero ellos siguen en su propia casa; no se los prueba de la misma manera que a las mujeres como nuevos integrantes de la familia. En ocasiones los conflictos no son superados y las mujeres deben regresar a sus hogares de origen. En caso de existir hijos, el ideal es que se queden en casa de su padre pero también pueden establecer arreglos de manutención y dejarlos con su madre. Los argumentos tanto del novio como de su familia refieren a lo poco trabajadora y poco respetuosa que fue la mujer. Desde el punto de vista de la mujer es que no congeniaron con la familia del novio y que ésta tiene costumbres distintas a las de su grupo doméstico.

La residencia virilocal de la unidad conyugal implica la salida de la mujer de su grupo familiar, lo que incide en la posición de las mujeres al interior de sus familias. Tanto las relaciones consanguíneas como las de alianza hacen que su situación sea ambigua. Como hija y hermana es percibida como "ajena", pues en algún momento se va para formar parte de otra unidad doméstica. Aunque esto le otorga, al mismo tiempo, cierta autonomía en su capacidad de decisión y de acumulación durante la etapa previa al matrimonio. Mientras a los varones, que permanecen en sus familias, se les socializa para el rol de dirigentes del espacio familiar y comunal.

Dado el carácter que asume el matrimonio en la organización social de la comunidad aymara las relaciones de género que se establecen en él son relevantes para entender la división del trabajo, el acceso a los factores productivos, la distribución y el consumo. Los hombres, situados en una posición de privilegio, controlan el trabajo de las mujeres al interior del hogar. Pero, la edad/generación resulta igualmente importante, ya que son las propias mujeres, en su condición de suegras, las que dirigen las labores femeninas en el período de mayor productividad y fertilidad. Así como el trabajo de los hombres es controlado por sus padres.

La tierra en manos de los hombres adultos no explica por sí misma el control del trabajo femenino. En las ciudades, donde la tierra deja de ser relevante y donde ambos aportan a los ingresos fa- 
miliares, la situación sigue siendo más o menos igual. Es en este sentido que el estatus femenino y las ideas respecto de la diferencia sexual, que definen a lo masculino y a los hombres como poseedores de mayor prestigio, ayudan a entender por qué la población masculina se beneficia gratuitamente del trabajo de las mujeres.

Los datos expuestos muestran un fuerte conflicto generacional que está afectando a las relaciones entre los géneros. Los hombres jóvenes se van desligando de las tareas propiamente campesinas por ser consideradas de menor prestigio. La educación de los hijos es pensada como una opción de ascenso social y cultural (ser menos "indios"). Si bien esta ausencia de los jóvenes provoca una sobrecarga en el matrimonio, es en las mujeres en quienes recae el mayor peso del trabajo. Los cambios que se van produciendo por efectos de la mayor mercantilización de la economía producen, por otra parte, una difícil situación para los hombres. La desvalorización de las tareas campesinas y la ausencia de oportunidades laborales para los jóvenes inciden en una desorientación para los mismos. Se agrega a ello la concentración de los medios de producción en los hombres mayores y la tendencia a preferir el trabajo por cuenta propia por sobre el trabajo dependiente.

A partir del rol que cumplen las mujeres en todas las fases del ciclo vital se podría decir que ellas son fundamentales en la sobrevivencia campesina. La preocupación de las jóvenes por acopiar lo necesario para su futuro hogar en la etapa previa al matrimonio resuelve en parte la subsistencia inicial del mismo, ya que su menor escolaridad las lleva a incorporarse a la producción de bienes antes que los hombres.

En resumen, la desigual distribución del trabajo, de los medios de producción y del consumo provoca tensiones en el hogar, particularmente en el matrimonio y en los hombres jóvenes. Situación que debe ser explicada tanto por los efectos de la desigualdad social en la que se encuentra la economía campesina en el orden regional y nacional, pero también porque las ideologías de género asignan a la mujer una posición de menor prestigio que los hombres y porque la sociedad aymara otorga mayor poder a los varones mayores. La edad o generación como canal de prestigio se combina con las ideas respecto al género en la teoría aymara de la división del trabajo. Quien tiene mayor autoridad en la familia es aquel que tiene más edad, in- dependientemente de su condición de género. A esto también se agregan las relaciones de parentesco o matrimonio. Son las mujeres-suegras las responsables de dirigir el trabajo de las mujeresesposas y son los hombres-padres quienes controlan a los más jóvenes.

Los antecedentes expuestos cuestionan, así, la reciprocidad y complementariedad supuestamente existente en la unidad "chacha-warmi" y afirmada en varios de los estudios regionales. A pesar de la flexibilidad del discurso y de las prácticas se dan relaciones de intercambio desiguales. Las mujeres en su condición de esposas asumen el rol de proveedoras, son las encargadas de la reproducción biológica, de la mano de obra y de la unidad social. Esta situación se agrava con el distanciamiento de los hombres de las tareas productivas. Sin embargo, las tendencias observadas en este estudio podrían ser erráticas al no considerar en el análisis dos dimensiones de gran relevancia: la composición de los ingresos campesinos y la diferenciación social al interior de las comunidades del altiplano. Ciertamente, la obtención diferencial de ingresos en el hogar, particularmente el que aportan los hombres vía venta de mano de obra, comercio o transporte, podría ser determinante. Por otro lado, el tipo de relaciones que se establecen dentro del hogar dependerá de su posición de clase. Las familias con mayores capacidades económicas pueden contratar mano de obra para una parte de los procesos de trabajo aquí identificados y de este modo la mujer-esposa o el hombre-esposo podrían liberarse. Aun así, los resultados de este estudio permiten concluir que las desigualdades sociales que genera la subordinación de la economía de los hogares altiplánicos a la economía nacional y las orientaciones valóricas impuestas por el estado nacional han profundizado las desigualdades sociales entre mujeres y hombres aymara, creando tensiones familiares de difícil solución.

Agracedimientos: Agradezco especialmente a las familias aymaras que me cobijaron en sus hogares. Los comentarios de mis compañeras de trabajo: Ana María Carrasco, Claudia Echeverría y Caprice Tabilo fueron de mucha utilidad. Pablo Ospina, tuvo la gentileza de compartir reflexiones y aportar sugerencias. Este trabajo forma parte de los proyectos FONDECYT 1970047 y CONICYT para Estudios de Género EG 96942. 


\section{Referencias Citadas}

Anderson, J.

1990 Sistemas de género e identidad de mujeres en culturas marcadas del Perú. Revista Peruana de Ciencias Sociales Vol. 2, No 1: 77-113.

Butler, J.

2000 El marxismo y lo meramente cultural. New Left Review 2: $109-122$.

Casos, V.

1990 La Mujer Campesina en la Familia y la Comunidad. Flora Tristán, Lima.

Carrasco, A.M.

1993 Participación social de las mujeres aymaras. Manuscrito en poder de la autora.

Carrasco, A.M.

1994 Mujeres aymaras y trabajo remunerado. Temas Regionales 1: 30-41.

Deere, C.D.

1992 Familia y Relaciones de Clase. El Campesino y los Terratenientes en la Sierra Norte del Período 1900-1980. Instituto de Estudios Peruanos, Lima.

De la Cadena, $M$.

1985 La comunera como productora. Allpanchis 25: 3-15.

De la Cadena, $M$.

1992 Las mujeres son más indias. Etnicidad y género en una comunidad de Cuzco. Espejos y travesías. Antropología y Mujer en los 90, editado por S. Montecinos y R. Rodríguez, pp. 16: 25-45. Isis Internacional, Santiago.

Flores, G.

1996 Desarrollo y mujer rural. Ponencia Presentada en el Seminario Mujer Rural, Arica.

Gavilán, V.

1994 Aportes para el análisis de la realidad rural de la Primera Región desde una perspectiva de género. Ponencia Presentada en el Seminario Taller La Situación Laboral de la Mujer en Chile, Santiago.

Gavilán, V.

1995 Una aproximación a las relaciones de género entre los aymaras del norte de Chile. Temas Regionales 2: 21-34.

González, H.

1990 Timar diagnóstico socioeconómico. La utilización de dos espacios económicos: el campo y la ciudad. Documentos de Trabajo 1-36. Taller de Estudios Andinos, Arica.

González, H. y H. Gundermann

1989 Campesinos y aymaras del norte de Chile. Documento de Trabajo 1: 3-30. Taller de Estudios Andinos, Arica.
Gundermann, $\mathrm{H}$.

1986 Comunidades ganaderas, mercado y diferenciación interna en el altiplano chileno. Chungara 16/17: 233-250.

Harris, O.

1980 The power of signs: gender, culture and the wild in the Bolivian Andes. En Nature, Culture and Gender, editado por C. Macormack y M. Strathern, pp. 70-94. Cambridge University Press, Cambridge.

Harris, O.

1985 Una visión andina del hombre y la mujer. Allpanchis 25: $17-42$.

Harris, O.

1986 La unidad doméstica como una unidad natural. Nueva Antropología 30: 199-222.

Harris, O. y K. Young

1981 Engendered structures: some problems in the analysis of reproducction. En The Anthropology of Precapitalist Societies, editado por J.S. Kahn y J.R. Llobera, pp. 109147. Macmillan Press, London.

Mackintosh, M.

1979 Domestic labour and the household. En Fit Work for Women, editado por S. Burman y S.T. Martines, pp. 173191. St. Martin's Press, New York.

Mackintosh, M.

1981 Gender and economics. the sexual division of labour and the subordination of women. En Of Marriage and the Market: Women's Subordination Internationally Perspective, editado por K. Young, C. Wolkowitz y R. McCullagh, pp. 1-15. CSE Books, London.

Moore, $\mathrm{H}$.

1990 Feminismo y Antropología. Ediciones Cátedra, Barcelona.

Pizarro, L.

1987 Algunas reflexiones acerca de los espacios femeninos y masculinos en las comunidades aymaras del sector de Isluga. Documento de Trabajo 10: 1-16. Taller de Estudios Regionales, Iquique.

Stolen K.A.

1987 A Media Voz: Ser Mujer Campesina en la Sierra Ecuatoriana. CEPLAES, Quito.

Van Kessel, J.

1992 Holocausto al Progreso. Hisbol, La Paz.

Warren K.B. y S.C. Bourque

1976 Campesinas y comuneras: subordinación sexual en la sierra peruana. Estudios Andinos 5: 77-97.

\section{Notas}

1 Las comunidades tienden a ser endogámicas, aunque esta práctica ha ido flexibilizándose de manera diferencial. Un ejemplo de ello lo observamos en la comunidad de Isluga donde el porcentaje de matrimonios endogámicos alcanza el 85\%; mientras en Cariquima sólo el 68,1\% (Gavilán 1995).

2 Durante mi residencia en Colchane, estancia de Isluga, presencié en muchas ocasiones a padres solicitando permiso para retirar a los hijos e hijas del internado o excusándose por sus ausencias debido a las necesidades de trabajo en tiempos de cosecha.

3 En la generación de hombres adultos éstos presentan mayores niveles de escolaridad y son las mujeres adultas quienes tienen los más altos niveles de analfabetismo.

4 Los testimonios indican que las mujeres lo hacían bajo la forma de empleo doméstico, en las actividades agrícolas y especialmente como tejedoras. 
5 El beneficio de la quinua o juira implica eliminar la cáscara amarga del grano para dejarla comestible; lo que se realiza a través de un largo proceso artesanal: tostando, pisando con los pies para desprenderla y luego se ventea.

6 Durante mi residencia más larga en Isluga (entre 1981 y 1983) era común ver a hombres y a mujeres adultos y jóvenes con sus carretillas camino al río a lavar. Hoy, sin embargo, los hombres van delegando esta tarea a la mujer.

7 Esta forma de aproximarnos a la intensidad del trabajo invertido por los miembros de una unidad doméstica se basó tanto en los registros etnográficos realizados durante el trabajo de campo como en las trayectorias laborales extraídas en las historias de vida y en las entrevistas aplicadas para confirmar estas interpretaciones.

$8 \quad$ El testimonio de F. Challapa refiriéndose al pensamiento de su marido ilustra bien lo que creen la mayoría de los padres: "Mis guaguas no van a ser como nosotros, van a educarse; por eso no los quiere mandar ni al llamo ni al cordero. Tienen que mirar sus cuadernos, sus libros, así. Mi marido llegó hasta $4^{\circ}$ año, se salió, ¿acaso niña es?, no pu'. Ahora los niños terminan curso. Las niñas primero así eran, pa' pastear cordero, llama, pa' eso no más querían, muy ignorante eran. Por eso yo pelo a mi abuelita. Nosotros tonto, tonto éramos, pensaba mejor me voy a salir de la escuela, pa' que esto. Así mismo todavía están algunos también. Yo le dejo que estudie mis niños, que termine su estudio."

9 Durante la década de los 80 varios de los jóvenes que terminaron la enseñanza media en Iquique retornaron a sus comunidades para dedicarse a las tareas campesinas, pero se orientaron principalmente al comercio.

10 En varias ocasiones me tocó presenciar demandas de mujeres jóvenes en contra de sus parejas por pago al trabajo de pastoreo realizado a las familias del novio durante el período de permanencia en la etapa previa al matrimonio.

11 Una anciana viuda me expresó su preocupación por la cantidad de objetos que dejaría como herencia a sus hijos e hijas. Pensaba que una de las formas de disminuir los conflictos entre ellos a la hora de la repartición era dejar una cantidad igual de frazadas de los distintos tipos, de manera que recibieran lo mismo. 